\title{
MUCOSAL LEISHMANIASIS A RARE ENTITY
}

Ritesh Shelkar ${ }^{1}$, Vipin Ekhar ${ }^{2}$, Adharsh Anand ${ }^{3}$, Sachin Rane ${ }^{4}$, Randhir Ghorpade ${ }^{5}$

\section{HOW TO CITE THIS ARTICLE:}

Ritesh Shelkar, Vipin Ekhar, Adharsh Anand, Sachin Rane, Randhir Ghorpade. "Mucosal Leishmaniasis a Rare Entity". Journal of Evolution of Medical and Dental Sciences 2014; Vol. 3, Issue 14, April 07; Page: 3614-3617, DOI: $10.14260 /$ jemds/2014/2330

ABSTRACT: Leishmaniasis is rare in India except some endemic areas in Orissa, Bihar etc. Although cutaneous and mucocutaneous varieties exist, pure mucosal varieties have been reported very rarely and such an entity has been questioned. But since the spread of AIDS, lots of atypical presentations of various diseases have been reported .Here we report a case of a 27 year old 7 months pregnant female, from Gondia, Maharashtra came with presenting complaints of left buccal mucosal ulcer since 3 months and left sided neck swelling since 2 months. As a routine protocol we checked her serostatus before a biopsy and was found to be HIV ELISA positive. Reports of FNAC from lymph nodal swelling, punch biopsy from ulcerative lesion and cervical lymph node excision biopsy were all consistent with positive for amastigote forms of leishmaniasis. We treated her with inj. sodium stibogluconate and HAART with complete resolution of lesions and improvement in CD4 counts.

KEYWORDS: Mucosal leishmaniasis, atypical presentations in HIV patients, amastigote forms

INTRODUCTION: Leishmaniasis is rare in India except some endemic areas in Orissa, Bihar etc. Although cutaneous and mucocutaneous varieties exist, pure mucosal varieties have been reported very rarely and such an entity has been questioned. Philip A Van Damme ${ }^{1}$ and colleagues had reported a rare case of mucosal leishmaniasis. Later Milian ${ }^{2}$ and colleagues reported a case of oral leishmaniasis from Spain where a hard vegetating growth was found on the hard palate. Though New World species of Leishmania are the main cause of mucocutaneous leishmaniasis, L donovani has rarely been incriminated in causation of mucosal involvement especially reported in Sudan. It has been seen that the mucosal leishmaniasis of Sudan, unlike South American mucocutaneous leishmaniasis, is not associated with cutaneous lesions. ${ }^{3}$ From India, one case of mucosal leishmaniasis was reported by Singh which was in an AIDS patient. ${ }^{4}$ This report emphasizes the need for specialists and clinical pathologists to rule out the diagnosis of mucosal leishmaniasis in Indian HIV positive patients, in whom it may be the first manifestation of their HIV status.

CASE REPORT: A 27 year old 7 months pregnant female, from Gondia, Maharashtra came with presenting complaints of left buccal mucosal ulcer since 3 months and left sided neck swelling since 2 months. She gave a history of loss of weight since last 6 months and chronic productive cough since 4 weeks. She had no history of any previous blood transfusion or high risk behavior.

Her general condition was moderate, thin frail and emaciated and her pulse was 88 per minute and BP was 110/60 mm of Hg. Pallor was present and there was no icterus, cyanosis, clubbing or edema. Left sided cervical lymphadenopathy was present.

In the left gingivobuccal sulcus there was a $7 \times 4 \mathrm{~cm}$ sub mucosal proliferative lesion involving the retromolar trigone area and the adjacent buccal mucosa with scattered ulcerated areas which was indurated and mildly tender (Figure 1). In the neck there was cervical lymphadenopathy 
involving the levels I, II, III, IV and V regions which was a single nodular hard non tender mass of size $10 \times 10 \mathrm{~cm}$ and skin over the mass was pinchable but the mass was fixed (Figure-2).

Her hematological reports showed a picture of pancytopenia and her sputum reports was normal. Before proceeding to a biopsy we did her ELISA test for HIV and was found positive and her CD4 count was 71/cub mm $(<100)$ and was diagnosed AIDS. Her cytology (FNAC from lymph nodal lesion) and biopsy reports (both punch biopsy of the mucosal lesion and cervical lymph nodal biopsy) showed amastigote forms of leishmaniasis organism (Figure-3).

Her bone marrow aspirate also showed amastigote forms of the same organism and she was positive for anti-rK- 39 antibody. Mean-while she developed preterm labor and resulted in a neonatal death.

We started her on sodium antimony stibogluconate injections and HAART. The injections were continued for 25 days and she responded well. The oral lesion resolved and there was significant decrease in the size of the neck swelling and her FNAC from the node didn't show any organism after 4 weeks of treatment. Her CD4 count improved (620/cub mm at 4 weeks) and she was discharged and kept in follow up.

DISCUSSION: Leishmaniasis is a parasitic disease caused by a haemoflagellate Leishmania. There are more than 21 species causing human infection. The infection is transmitted to humans through the bites of female sandflies (Figure-4) belonging to 30 different species. Depending on the species, it can manifest as Cutaneous Leishmaniasis (CL), Mucocutaneous Leishmaniasis (MCL), Diffused Cutaneous Leishmaniasis (DCL) or Visceral Leishmaniasis (VL). Leishmaniasis is prevalent in at least 88 countries. More than 90 percent of the cutaneous cases occur in Afghanistan, Saudi Arabia, Algeria, Brazil, Iran, Iraq, Syria and Sudan; while more than 90 percent of visceral cases occur in India and Sudan. Mucocutaneous form is mostly found in Latin America. ${ }^{5,6}$ and mucosal forms are very rarely reported.

Approximately 350 million people live in the area of active parasite transmission. Though several animal reservoirs have been identified in other endemic countries, no animal reservoir of the parasite has yet been identified in India. It is believed that skin lesions of a late sequel of the visceral form called post-kala azar- dermal leishmanisis (PKDL) act as reservoirs. ${ }^{5,6}$

HIV co infection can result in the atypical presentations of the disease. In our case the patient presented with a gingivobuccal sulcus lesion and cervical lymphadenopathy which may direct the clinician to the diagnosis of an oral malignancy and moreover she was not a diagnosed case of HIV and this was her first presentation. Her positive sero status and more focused clinical examination made us clear that it might be the presentation of an opportunistic infection as the lesion was submucosal and the lymphadenopathy was more of a firm rubbery consistency.

This report also emphasizes the need for specialists and clinical pathologists not to rule out the diagnosis of mucosal leishmaniasis in Indian HIV positive patients, in whom it may be the first manifestation of their HIV status.

\section{REFERENCES:}

1. Van Damme PA, Keuter M, van Assen S, De-Wilde PM, Beckers PJA. A rare case of oral leishmaniasis. Lancet Infect Dis 2004; 4: 53. 


\section{CASE REPORT}

2. Milian MA, Bagan JV, Jimenez Y, Perez A, Scully C. Oral leishmaniasis in a HIV-positive patient. Report of a case involving the palate. Oral Dis 2002; 8: 59-61.

3. El-Hassan AM, Zijlstra EE. Leishmaniasis in Sudan: mucosal leishmaniasis. Trans R Soc Trop Med Hyg 2001; 95 (suppl 1): S19-26.

4. Singh S, Sivakumar R. Recent advances in the diagnosis of leishmaniasis. J Postgrad Med 2003; 49:55-60.

5. Herwaldt BL. Leishmaniasis. Lancet. 1999; 354:1191-9.

6. Geographical Distribution of Leishmaniasis: http://www.who.int/emc/ diseases/leish/ leisgeo.html.

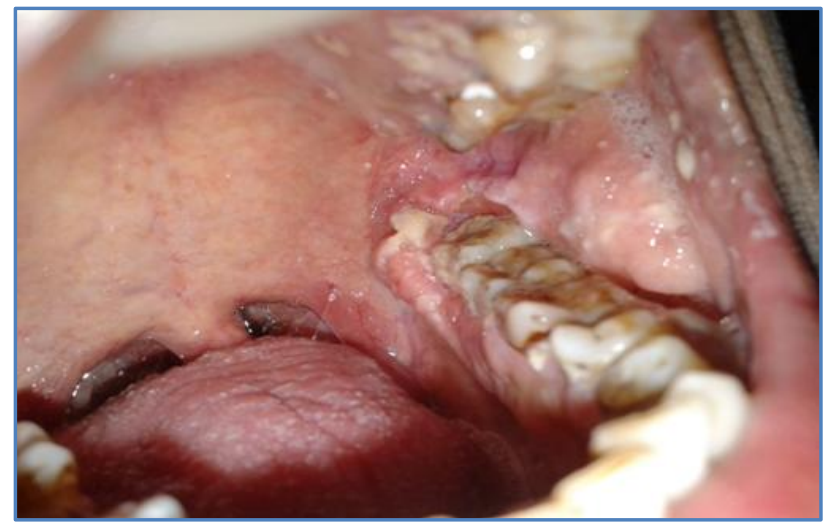

Figure 1

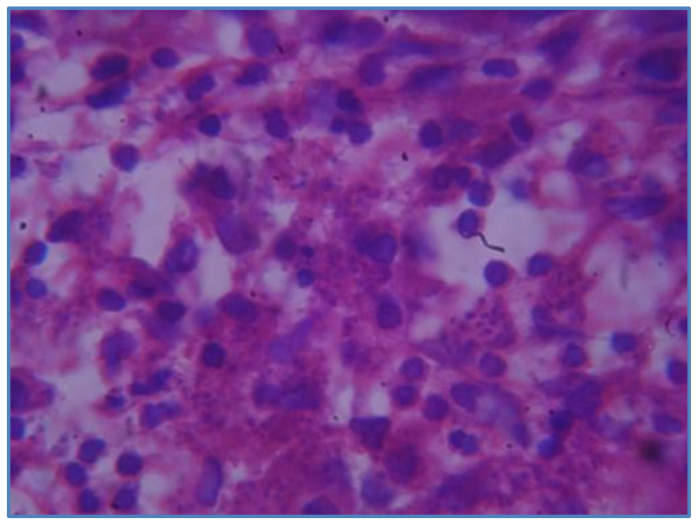

Figure 3

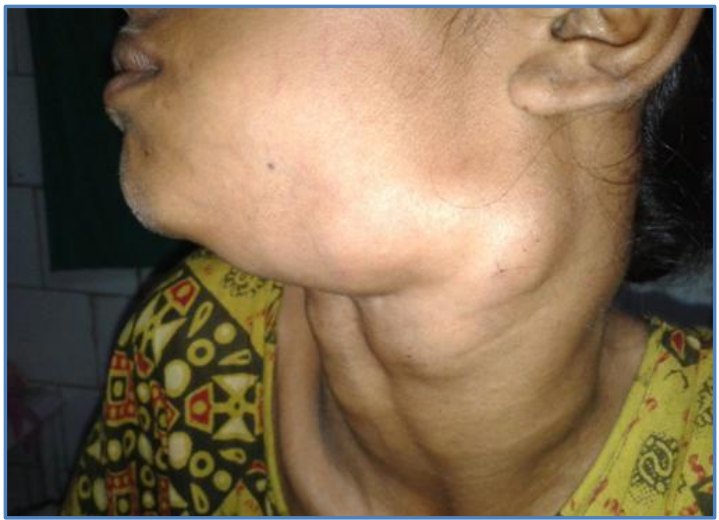

Figure 2

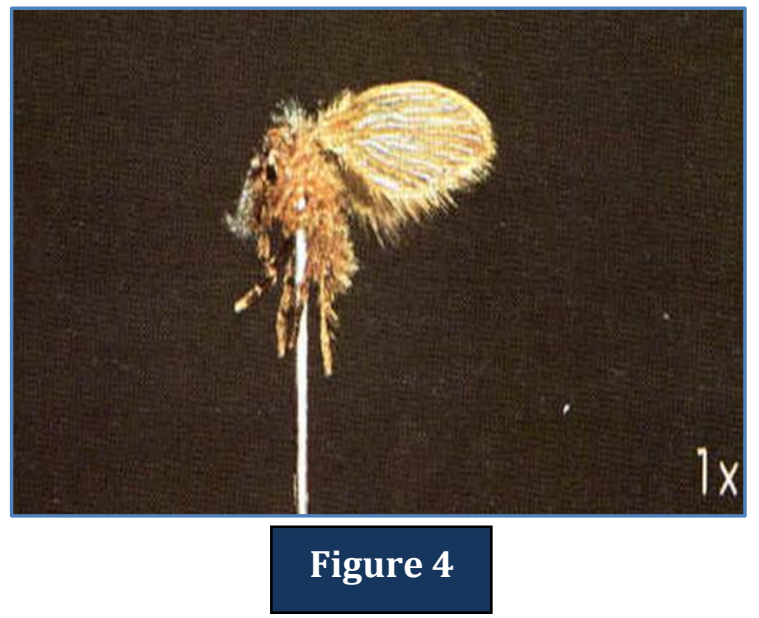




\section{CASE REPORT}

\section{AUTHORS:}

1. Ritesh Shelkar

2. Vipin Ekhar

3. Adharsh Anand

4. Sachin Rane

5. Randhir Ghorpade

\section{PARTICULARS OF CONTRIBUTORS}

1. Assistant Professor, Department of ENT, Indira Gandhi Government Medical College, Nagpur, Maharashtra.

2. Associate Professor, Department of ENT, Indira Gandhi Government Medical College, Nagpur, Maharashtra.

3. Junior Resident, Department of ENT, Indira Gandhi Government Medical College, Nagpur, Maharashtra.
4. Junior Resident, Department of ENT, Indira Gandhi Government Medical College, Nagpur, Maharashtra.

5. Junior Resident, Department of ENT, Indira Gandhi Government Medical College, Nagpur, Maharashtra.

\section{NAME ADDRESS EMAIL ID OF THE CORRESPONDING AUTHOR:}

Dr. Ritesh Shelkar, \#230, Medical Road, Opposite Ambedkar High School, Untakhana, Nagpur- 440009, Maharashtra.

E-mail: riteshnshelkar@gmail.com

Date of Submission: 10/03/2014.

Date of Peer Review: 11/03/2014.

Date of Acceptance: 24/03/2014.

Date of Publishing: 01/04/2014. 